\title{
Kritische Instanz der deutschen und europäischen Umweltpolitik
}

\author{
Der Sachverständigenrat für Umweltfragen \\ ist eine der ältesten Einrichtungen zur Unter- \\ stützung der Umweltpolitik in Deutschland. \\ Manchmal ist er seiner Zeit voraus, oft \\ verschafft er sich Gehör, selten wird er \\ ignoriert. Zum Wohl des Menschen und \\ der Umwelt arbeitet er immer. \\ Von Christian Hey
}

D er Sachverständigenrat für Umweltfragen (SRU) wurde am 28. Dezember 1971 eingerichtet. Seine Einrichtung war damit noch lange vor dem Umweltbundesamt und der Einrichtung eines Umweltministeriums eine der ersten Institutionenbildungen der neueren deutschen Umweltpolitik. Gemäß dem Einrichtungserlass ist die zentrale Aufgabe des SRU die „periodische Begutachtung der Umweltsituation und Umweltbedingungen“ und die „Erleichterung der Urteilsbildung bei allen umweltpolitisch verantwortlichen Instanzen sowie in der Öffentlichkeit“.

Der SRU soll „Fehlentwicklungen und Möglichkeiten zu deren Vermeidung oder zu deren Beseitigung“ aufzeigen. Mit dieser Aufgabe werden sieben für vier Jahre von der Bundesregierung ernannte Universitätsprofessoren aus unterschiedlichen umweltbezogenen Fachdisziplinen betraut, die in ihrer wissenschaftlichen Arbeit von einer Geschäftsstelle unterstützt werden. Die sieben Professoren sind jeweils anerkannte Kapazitäten in ihrem Fach.

\section{Freiräume zur Meinungsbildung}

Das Mandat des Erlasses ist breit und verschafft dem SRU erhebliche Freiräume zur selbstbestimmten Prioritätensetzung und Funktionsbestimmung. Das Mandat geht über die unmittelbare Beratung der für Umweltpolitik zuständigen Bundesressorts hinaus. Der SRU ist eine Instanz der öffentlichen Meinungsbildung und damit letztlich der Demokratisierung der Umweltpolitik, da er fachöffentliche Informationen allgemeinöffentlich zugänglich macht sowie von unabhängiger und fachlich hochkompetenter Perspektive bewertet.

Seine Gutachten sind damit oft ein zentraler Referenzpunkt in aktuellen Debatten. Er hat die Freiheit, auch politisch unangenehme Wahrheiten auszusprechen. Die Bundesregierung leistet sich damit eine Instanz der kritischen Bewertung getrof- fener Maßnahmen und des wissenschaftsgestützten, konzeptionellen Anstoßens neuer umweltpolitischer Ansätze und Maßnahmen. In den über drei Jahrzehnten seines Bestehens hat der SRU versucht, das Mandat in aller Breite auszufüllen. Der periodischen Berichterstattungspflicht kommt er mit seinen alle vier Jahre erscheinenden Umweltgutachten nach. Diese bilden eine detaillierte Gesamtevaluation der Umweltpolitik. Durch die jeweils einem großen Problemfeld gewidmeten Sondergutachten erfüllt der SRU seine Funktion des konzeptionellen Anstoßens von Politik. Hierbei formuliert er regelmäßig ein komplexes Handlungsprogramm. Eher der aktuellen politischen Meinungsbildung oder unmittelbaren Politikberatung sind die circa vier Mal jährlich erscheinenden aktuellen Stellungnahmen gewidmet.

\section{Bedeutung des SRU}

Der SRU ist zu einem Zeitpunkt gegründet worden, als der Glaube in das „technisch-rationale“ Modell wissenschaftlicher Politikberatung noch relativ ungebrochen war und es einer Wissenschaftsinstanz bedurfte, die der Politik den Stand der wissenschaftlichen Erkenntnis übersetzte. Mittlerweile sind sowohl das technisch-rationale Ursprungsmodell einer auf objektiven, wissenschaftlichen Erkenntnis fußenden Politikberatung als auch das einer einzigen Beratungsinstanz überholt. Im Zeitalter unstrukturierter Probleme, schwer determinierbarer Risiken und kontrovers diskutierter Lösungsvarianten gibt es kein objektiv richtiges Wissen mehr. Auch hat sich die wissenschaftliche Beratungslandschaft pluralisiert, ausgeweitet und themenspezifisch ausdifferenziert. Der SRU steht damit im Konzert oder im Wettbewerb zu anderen wissenschaftlichen Rat- und Ideengebern. Dennoch hat er eine besondere Stellung in der ausdifferenzierten Beratungslandschaft:

- Die Arbeit des SRU ist weder Auftragsforschung noch akademische Grundlagenforschung. Sie ist orientierungsgebende, informierende und bewertende Synthese des aktuellen fachlichen Diskussionsstandes und hat damit eine wichtige Bündelungsaufgabe, die ansonsten nicht wahrgenommen werden könnte.

- In dieser Funktion bildet die Tätigkeit des SRU eine Brücke zwischen Wissenschaft und Politik. Die Ergebnisse müssen sowohl in der jeweiligen wissenschaftlichen Fachwelt vertretbar als auch als Rat für die Umweltpolitik relevant sein. Für die Qualität der Arbeit stehen die jeweiligen Ratsmitglieder mit ihrem Ruf ein. Dieser im universitären Wissenschaftssystem und in der Fachwelt rückgekoppelte Rat ist die Quelle der Autorität und Glaubwürdigkeit des SRU. 
- Die Gutachten bilden eine wichtige Orientierungsmarke für die Umweltverwaltung und Verbände sowie zur Untermauerung von deren Anliegen, nicht selten auch für das Wissenschaftssystem selbst. Der SRU kann damit auch eine wichtige Schiedsrichterfunktion in umweltpolitischen Kontroversen ausüben, an vergessene Probleme erinnern, Hinweise zum effizienteren Einsatz umweltpolitischer Instrumente geben sowie bestimmte Ansätze und Maßnahmen delegitimieren.

I Dank seiner multidisziplinären Zusammensetzung ist er in der Lage, sowohl das eher naturwissenschaftliche Wissen für den ökologischen Problemdiskurs als auch das eher gesellschaftswissenschaftlich und technische Wissen zur Debatte um Lösungen auszuwerten. Der ökonomischer Rat des SRU reflektiert zum Beispiel sowohl den ökologischen Handlungsbedarf als auch rechtlich-institutionelle Barrieren und kann damit eine größere Wirkung entfalten als eine rein theoriegeleitete Politikempfehlung.

\section{Erfolgsbedingungen}

In den letzten Jahrzehnten oszillierte das Selbstverständnis des SRU immer wieder zwischen der Akzentuierung auf grundsätzliche Orientierungen, Ideen und Paradigmen und der auf die unmittelbare Politikberatung. Mit der klaren Produktdifferenzierung des SRU werden nunmehr beide Ziele verfolgt: Die Grundsatzkapitel der Umweltgutachten und die Sondergutachten versuchen eher die langsame Diffusion grundlegender Ideen in der Umweltdiskussion zu erreichen. Beispiele dafür sind der Nachhaltigkeits- und Mobilitätsbegriff des SRU oder die Ausrichtung der Umwelt- und Verkehrspolitik auf einen qualitätsorientierten Steuerungsansatz. Die aktuellen Stellungnahmen wie etwa zur Verbandsklage, zur Feinstaubdebatte oder zur Klimapolitik setzen Akzente in einer aktuellen umweltpolitischen Auseinandersetzung.

Der Einfluss des SRU auf grundsätzliche Orientierungen ist schwer zu messen, aber nicht zu unterschätzen. Neue Ideen stoßen zumeist erst einmal auf starke Widerstände, benötigen lange Vorlaufzeiten um breite Akzeptanz zu finden und werden zumeist nicht nur von einem Akteur entwickelt und vorgebracht. Die Urheberschaft ist daher schwer zu rekonstruieren. Erfolgreich ist der SRU in dieser Hinsicht, wenn er auf Resonanz stößt, wenn sich andere Akteure explizit oder implizit auf den SRU beziehen und damit die Ideen weitere Kreise ziehen. Der derzeitige agrarpolitische Reformprozess hin zur differenzierten Honorierung ökologischer Leistungen ist vom SRU bereits Mitte der 80er Jahre gefordert worden. Der in den Gutachten von 2002 und 2004 geforderte Paradigmenwechsel in der Abfallpolitik weg von einer pauschalen Vermutung der Höherwertigkeit der Verwertung, hin zur Formulierung ökologischer Rahmenbedingungen scheint von der Europäischen Kommission wesentlich schneller übernommen worden zu sein. In beiden Fällen gibt der neue Ansatz eine bessere Antwort auf gravierend gewordene Akzeptanz- oder Effizienzprobleme bisheriger Politiken und fügt sich besser in einen neuen allgemeinen Ordnungsrahmen ein. Ein außerordentliches Erfolgsbeispiel ist auch die Forderung des SRU nach einer nationalen Naturschutz- oder Biodiversitätsstrategie, um die sich ein breites Bündnis des Naturschutzes geschart hat. Hoffentlich wird sie in Zukunft auch als Teil der nationalen Nachhaltigkeitsstrategie durch das BMU umgesetzt. In diesem Fall wäre die breite Zustimmung zu einem moderneren Naturschutzverständnis in der Fachwelt und dem BMU die zentrale Erfolgsbedingung.

\section{Resonanz in der Tagespolitik}

Der tagespolitische Erfolg von Ratschlägen des SRU ist hingegen in Form der unmittelbaren Resonanz von Medien, Politik, Fachwelt und manchmal auch in Form von Politikänderungen leichter zu messen, So waren sicher die frühen Zwischenrufe des SRU bei der Novellierung des Baugesetzbuches oder der Meeresraumplanung im Jahr 2003 ebenso hilfreich und direkt sichtbar, wie sein Werben für ambitionierte Klimaschutzziele und die Fortsetzung einer Vorreiterrolle im Klimaschutz gegen massive Angriffe großer Industrieverbände.

Die neueste Koalitionsvereinbarung zum Klimaschutz spiegelt in auffälliger Weise Argumentationsmuster einer entsprechenden aktuellen Stellungnahme des SRU zu diesem Thema vom Herbst 2005 wider, die auf beträchtliche Medienresonanz gestoßen ist. Medienresonanz ist dabei aber nicht mit Einfluss gleichzusetzen. So manche Äußerung des SRU mit großer Medienresonanz, wie etwa zum so NAP II, schlug sich nicht in sichtbarem Politikwandel nieder und umgekehrt.

Der SRU hat in weniger politisierten und polarisierten Feldern, in sehr umweltfachlich geprägten Gebieten wie Aerosolemissionen von Kompostierungsanlagen oder dort, wo die Überschneidung wirtschafts- und umweltpolitischer Anliegen betont wird, mehr Einfluss. Weniger Einfluss besteht dort, wo massive ökonomische Interessen oder eine bereits fest entschiedene Politik infrage gestellt werden. Der richtige Zeitpunkt ist ebenfalls eine wichtige, aber nicht immer erreichbare Erfolgsbedingung für die Empfehlung des SRU: Das Entscheidungssystem muss noch beratungsoffen sein.

\section{Literatur}

Hey, C.: Umweltberatung und Politik: Das Beispiel des Sachständigenrates für Umweltfragen. In: ZAU Sonderheft 15/2005, S. 55-68.

Owens, S./ Rayner, T.: When Knowledge Matters: The Role and Influence of the Royal Commission on Environmental Pollution. In : Journal of Environmental Policy Planning. 1/ 1999, pp. 7-24.

Ziegler, H.: SRU und WBGU - die Umweltpolitikberatung der Bundesregierung. In: Jahrbuch Ökologie 2004. München 2004, S. 80-93.

\section{AUTOR + KONTAKT}

Dr. Christian Hey ist Generalsekretär des Sachverständigenrates für Umweltfragen (SRU).

SRU, Reichpietschufer 60, 10785 Berlin. Tel: 030/ 263696-0,E-Mail: sru-info@uba.de

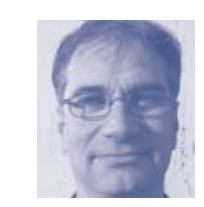


(c) 20I0 Authors; licensee IÖW and oekom verlag. This is an article distributed under the terms of the Creative Commons Attribution Non-Commercial No Derivates License (http://creativecommons.org/licenses/by-nc-nd/3.o/), which permits unrestricted use, distribution, and reproduction in any medium, provided the original work is properly cited. 